\title{
POWERS OF IDEALS GENERATED BY QUADRATIC SEQUENCES
}

\author{
K. N. RAGHAVAN
}

\begin{abstract}
Huneke's conjecture that weak $d$-sequences generate ideals of quadratic type is proved. The proof suggests the definition of quadratic sequences, which are more general than weak $d$-sequences yet simpler to define and han$\mathrm{dle}$, in addition to being just as useful. We extend the theory of $d$-sequences and weak $d$-sequences to quadratic sequences. Results of Costa on sequences of linear type are generalized. An example of a two-dimensional local domain in which every system of parameters is a $d$-sequence in some order but which nevertheless fails to be Buchsbaum is given. A criterion is established for when equality holds in Burch's inequality for an ideal generated by a quadratic sequence.
\end{abstract}

\section{INTRODUCTION}

In this paper, we simplify and extend the theory, due to Huneke (see [H1, $\mathrm{H} 2, \mathrm{H} 3]$ ), of $d$-sequences and weak $d$-sequences. Brodmann [Br1] showed that for an ideal $J$ of a Noetherian ring $R$, the value of $\operatorname{depth} R / J^{n}$ (with respect to any ideal) is independent of $n$ for large $n$. But calculating this asymptotic value of depth for a given ideal is a difficult problem. Huneke [H1] introduced the concept of $d$-sequence in order to calculate this asymptotic value for specific examples of ideals $J$. Since then, the centrality of the notion of $d$-sequence has been realised, thanks to the work of several authors.

An ordered sequence $x_{1}, \ldots, x_{n}$ of a ring $R$ (all rings are commutative with identity) is said to be a $d$-sequence if either (both) of the following equivalent conditions hold:

(1) $\left(\left(x_{1}, \ldots, x_{i-1}\right): x_{i} x_{k}\right)=\left(\left(x_{1}, \ldots, x_{i-1}\right): x_{k}\right)$ for all $1 \leq i \leq n$ and for all $k \geq i$

(2) $\left(\left(x_{1}, \ldots, x_{i-1}\right): x_{i}\right) \cap\left(x_{1}, \ldots, x_{n}\right)=\left(x_{1}, \ldots, x_{i-1}\right)$ for all $1 \leq i \leq n$. where $\left(x_{1}, \ldots, x_{i-1}\right)$ is interpreted as 0 when $i=1$. Regular sequences clearly are $d$-sequences. One example of a $d$-sequence that is not a regular sequence is provided by the maximal minors (in any order) of a generic $n \times(n+$ 1) matrix (see Example 4.1 of $\S 4$ ). There are many natural contexts in which $d$-sequences appear: see $\S 4$. Huneke later developed the theory of weak $d$ sequences and used it effectively to calculate the asymptotic value of depth $R / J^{n}$ for an even larger class of examples of ideals $J$ (see [H2]). As the terminology

Received by the editors August 29, 1991 and, in revised form, November 2, 1992.

1991 Mathematics Subject Classification. Primary 13C40, 13F50.

Key words and phrases. $d$-sequence, weak $d$-sequence, quadratic sequence, relation type, RatliffRush ideal, analytic deviation, Buchsbaum ring, Burch's inequality. 
suggests, $d$-sequences are weak $d$-sequences. The maximal minors of a generic $m \times n$ matrix form a weak $d$-sequence. More generally, the elements of a straightening-closed ideal of an ordinal Hodge algebra form a weak $d$-sequence (see $\S 4)$.

Recall the following well-known definition.

Definition 1.1. A relation on an ordered sequence of elements $x_{1}, \ldots, x_{n}$ of a ring $R$ is a form of positive degree in the graded polynomial ring $S=$ $R\left[U_{i} \mid 1 \leq i \leq n\right]$ (elements of $R$ have degree 0 and each $U_{i}$ has degree 1) that yields 0 when evaluated at $x_{1}, \ldots, x_{n}$. Let $\mathbf{N}_{j}$ denote the ideal of $S$ generated by the relations on $x_{1}, \ldots, x_{n}$ of degree at most $j$. We have an ascending chain $\mathbf{N}_{1} \subseteq \mathbf{N}_{2} \subseteq \cdots$ of ideals of $S$. Let $\mathbf{r}$ be the least positive integer (possibly infinity) such that $\mathbf{N}_{\mathbf{r}}=\bigcup_{j \geq 1} \mathbf{N}_{j}$. (If $R$ is Noetherian, then $r$ must be finite by Hilbert's basis theorem.) It can be shown, by elementary means, that $\mathbf{r}$ is independent of the choice of a finite generating set of the ideal $X=\left(x_{1}, \ldots, x_{n}\right)$. The invariant $\mathbf{r}$ is called the relation type of the ideal $X=\left(x_{1}, \ldots, x_{n}\right)$. An ideal of relation type 1 is said to be of linear type. An ideal of relation type at most 2 is said to be of quadratic type.

The following definition is of crucial importance in this paper.

Definition 1.2. Given a ring $R$, and a form $f$ of positive degree of the polynomial ring $S=R\left[U_{1}, \ldots, U_{n}\right]$ in $n$ variables over $R$, the order of $f$ is the least integer $w$ such that $f$ belongs to the ideal $\left(U_{1}, \ldots, U_{w}\right)$ of $S$ (the only form of order 0 is 0 ).

In [R], the author proved, using induction on the order of a certain polynomial, a theorem about $d$-sequences, from which important results about $d$ sequences can be deduced as corollaries (see also [R1]). The notion of order of an element of a polynomial ring has other useful applications. In $\S 2$, we obtain generalizations of results of Costa [C], and in $\S 3$, we prove Huneke's conjecture that weak $d$-sequences generate ideals of quadratic type (see Definition 1.1). (In the proof of Huneke's conjecture, the notion of order is replaced by the more general notion of an ideal (of a poset) dividing a homogeneous element of the polynomial ring.) The proof of the main Theorem 3.6 of $\S 3$ suggests the definition of quadratic sequences. These are not only easier to define and more general than weak $d$-sequences, but also, as we show in $\S 5$, the whole theory of weak $d$-sequences can be extended to these sequences. The definition of quadratic sequences is cleaner and more natural than that of weak $d$-sequences. It also suggests the definition of "tertiary sequences", etc. In $\S 3$, we also show that in a domain, ideals generated by quadratic sequences are Ratliff-Rush (see Definition 3.9).

In the Main Theorem 5.4 of $\S 5$, we show that cyclic modules defined by the powers of an ideal generated by a quadratic sequence can be filtered by ideals that are "related" to the quadratic sequence. Our proof is simpler than Huneke's original proof of the same result for $d$-sequences and weak $d$-sequences.

Important special cases of our theorem that quadratic sequences generate ideals of quadratic type have been known. That straightening-closed ideals are of quadratic type follows from the graded ordinal Hodge algebra structure on their Rees algebras (see [BST, Lemma 2.2.1]). In $\S 4$, which is devoted to examples, we add to the list of weak $d$-sequences. Namely, we show that ideals 
of small analytic deviation studied recently by Huckaba and Huneke [HuH1, $\mathrm{HuH} 2$ ] are generated by weak $d$-sequences, hence by quadratic sequences. It follows that these ideals are of quadratic type. This recovers a result of [HuH1] for ideals of analytic deviation 1. For ideals of analytic deviation 2, this is a new result. Morales and Simis [MS] and Schenzel [S] have shown that defining ideals of monomial curves in $\mathbb{P}^{3}$ that lie on the quadric surface $x y-z w=0$ are of quadratic type; these ideals are generated by weak $d$-sequences (see, for example, [MS]). Since these ideals have analytic deviation 1 and are special cases of the ideals studied by Huckaba and Huneke, we recover also this result.

In $\S 6$ we give an example of a two-dimensional Noetherian local domain in which every system of parameters is a $d$-sequence in some order, but which nevertheless is not Buchsbaum. This is interesting because a Noetherian local ring is Buchsbaum if and only if every system of parameters is a $d$-sequence. In $\S 7$ we establish a sufficient criterion for equality to hold in Burch's inequality for an ideal generated by a quadratic sequence. This is an extension of a theorem of Huneke for $d$-sequences. Our proof is simpler than Huneke's proof and even enables us to weaken his hypothesis.

\section{SEQUences that EFFECTIVELy BIND RELATION TYPE}

In this section, we generalize results of $\S 1$ of Costa's paper [C]. We also give counterexamples to two questions raised in [C]. Fix notation as below.

Notation. Given an ordered sequence $x_{1}, \ldots, x_{n}$ of elements of a ring $R$, we denote the ideal $\left(x_{1}, \ldots, x_{n}\right)$ by $X$, and the partial ideal $\left(x_{1}, \ldots, x_{i}\right)$ by $X_{i}$. It is convenient to let $X_{0}=0$. The graded polynomial ring $R\left[U_{i} \mid 1 \leq i \leq n\right]$ in $n$ indeterminates over $R$ is denoted by $S$; elements of $R$ have degree 0 and each $U_{i}$ has degree 1 . For an element $f$ of $S$, we denote by $f(\underline{x})$ the image of $f$ in $R$ under the evaluation map from $S$ to $R$ defined by $U_{i} \mapsto x_{i}$.

Consider the following conditions on the sequence $x_{1}, \ldots, x_{n}(p$ denotes a positive integer) :

$$
\begin{array}{cc}
\left(X_{i-1} X^{p-1}: x_{i}\right) \cap X^{p}=X_{i-1} X^{p-1} & \forall 1 \leq i \leq n, \\
\left(X_{i-1} X^{p}: x_{i}\right) \cap X^{p}=X_{i-1} X^{p-1} & \forall 1 \leq i \leq n .
\end{array}
$$

Costa proves the following in $\S 1$ of $[\mathrm{C}]$ :

(1) If $(*)$ holds for $p=1$, then it holds for all $p \geq 1$.

(2) If $\left(^{*}\right)$ holds for all $p \geq 1$, then $X$ is of linear type.

(3) If ( $\dagger$ ) holds for all $p \geq 1$, then $X$ is of linear type.

Recall that $x_{1}, \ldots, x_{n}$ is called a $d$-sequence (by definition) if $\left(^{*}\right)$ holds for $p=1$. Thus it follows from (1) and (2) that if $x_{1}, \ldots, x_{n}$ is a $d$-sequence, then $X$ is of linear type. Since, for a fixed $p,(\dagger)$ is weaker than $\left(^{*}\right)$, it follows that $(3) \Rightarrow(2)$. It is easy to deduce (1) from the fact [H1, Theorem 2.1] that, if $x_{1}, \ldots, x_{n}$ is a $d$-sequence, then $X_{i-1} \cap X^{p} \subseteq X_{i-1} X^{p-1} \quad \forall 1 \leq i \leq n$ : just note that $\left(X_{i-1} X^{p-1}: x_{i}\right) \cap X^{p} \subseteq\left(X_{i-1}: x_{i}\right) \cap X^{p}=\left[\left(X_{i-1}: x_{i}\right) \cap X\right] \cap X^{p}=$ $X_{i-1} \cap X^{p}=X_{i-1} X^{p-1}$; the other containment holds in general.

Here we prove, from first principles, the following generalizations $(m$ denotes a fixed positive integer) :

$\left(1^{\prime}\right)$ If $\left(^{*}\right)$ holds for $p=m$, then it holds for all $p \geq m$. 
(2') If $\left(^{*}\right)$ holds for $p=m$, then the relation type of $X$ is at most $m$.

$\left(3^{\prime}\right)$ If $(\dagger)$ holds for $p=m$, then every relation (see Definition 1.1) of degree $(m+1)$ on $x_{1}, \ldots, x_{n}$ belongs to the ideal generated by relations of degree $m$.

The following example shows that the hypothesis of (3) above may be satisfied even though the hypothesis of $\left(2^{\prime}\right)$ may not be satisfied for any value of $m$.

Example 2.1. Let $R$ be the subring $k\left[x, x y, y^{3}, y^{4}\right]$ of the polynomial ring $k[x, y]$ over a field $k$. Consider the sequence $x, y^{4}$ in $R$. (The sequence $y^{4}, x$ is a $d$-sequence.) We claim that for this sequence ( $\dagger$ ) holds $\forall p \geq 1$ but $\left(^{*}\right)$ does not hold for any value of $p$. We first show the latter half of the claim. Since $y^{5} x \in\left((x): y^{4}\right) \cap\left(x, y^{4}\right) \backslash(x)$, it is clear that $\left(^{*}\right)$ does not hold for $p=1$. Let $p \geq 2$. Notice that if $\left(^{*}\right)$ holds, then $\left(X_{i-1} X^{p-1}\right.$ : $\left.x_{i}\right) \cap\left(x_{i+1}, \ldots, x_{n}\right)^{p} \subseteq X_{i-1} X^{p-1}$, which in turn would imply that $X_{i-1} X^{p-2} \cap$ $\left(x_{i+1}, \ldots, x_{n}\right)^{p} \subseteq X_{i-1} X^{p-1}$. But it is easy to see that $y^{4 p+1} x \in\left(x\left(x, y^{4}\right)^{p-2} \cap\right.$ $\left.\left(y^{4}\right)^{p}\right) \backslash\left(x\left(x, y^{4}\right)^{p-1}\right)$, as follows : notice that $y^{4 p+1} x=x y^{4(p-2)} \cdot y^{9}=\left(y^{4}\right)^{p} \cdot y x$, which shows that $y^{4 p+1} x \in x\left(x, y^{4}\right)^{p-2} \cap\left(y^{4}\right)^{p}$; since $y^{5} \notin R$, we see that $y^{4 p+1} x \notin x\left(x, y^{4}\right)^{p-1}$.

To see the first half of the claim, notice first that for $i=1$ both sides are equal to 0 so we need only show it for $i=2$. Using the modular law $A \cap(B+C)=(A \cap B)+C$ (where $A, B, C$ are submodules with $C \subseteq A$ ), we see that it is enough to show that $\left(x\left(x, y^{4}\right)^{p}: y^{4}\right) \cap\left(y^{4 p}\right) \subseteq x\left(x, y^{4}\right)^{p-1}$. For this it is enough to show that $\left(x\left(x, y^{4 p}\right): y^{4}\right) \cap\left(y^{4 p}\right) \subseteq\left(x y^{4(p-1)}\right)$. Let $a$ be an element belonging to the left side. Then $a=b y^{4 p}$ and $a y^{4}=c x^{2}+d x y^{4 p}$, where $b, c, d \in R$. Substituting the first equation into the second, we see that $x \mid b$ in the polynomial ring $S=k[x, y]$. Writing $b=b_{1} x$, we get $a=\left(b_{1} y^{4}\right)\left(x y^{4(p-1)}\right)$. Thus it is enough to show that $b_{1} y^{4} \in R$. Substituting the last expression for $a$ into the expression for $a y^{4}$ and cancelling $x$, we get $b_{1} y^{4(p+1)}=c x+d y^{4 p}$. But, since $y^{5} \notin R$, this is possible only if the coefficient of $y$ is 0 in the expression of $b_{1}$ as a sum of monomials in $S$. This shows that $y^{4} b_{1} \in R$.

Proposition 2.2. Let $m$ be a fixed positive integer, and let $x_{1}, \ldots, x_{n}$ be an ordered sequence of elements of a ring $R$ such that $\left({ }^{*}\right)$ holds with $p=m$. Let $i$ be an integer such that $1 \leq i \leq n$. Then, for any form $f$ of degree $p \geq m$ in the polynomial ring $S=R\left[U_{i} \mid 1 \leq i \leq n\right]$ such that $f(\underline{x})$ belongs to $X_{i-1} X^{m-1}$, there exists a form $g$ in $S$ of degree $p$ and order less than $i$ such that $f \equiv g$ modulo $\mathbf{N}_{m}$ where $\mathbf{N}_{m}$ denotes the ideal of $S$ generated by the relations of degree at most $m$ on the elements $x_{1}, \ldots, x_{n}$.

Proof. The proof is by a double induction, the first on $p$, the second on the order of $f$. First suppose that $p=m$. Since $f(\underline{x})$ belongs to $X_{i-1} X^{m-1}$, there exists a polynomial $g$ of degree $m$ and order less than $i$ such that $f(\underline{x})=g(\underline{x})$. Note that $f-g$ is a relation of degree $m$ on $x_{1}, \ldots, x_{n}$.

Now let $p>m$. Let $w$ denote the order of $f$ and proceed by induction on $w$. If $w<i$, then the statement is clear (take $g=f$ ). Assume that $w \geq i$. Write $f=U_{w} f_{1}+h$, where $f_{1}$ is a form of degree $p-1$ and $h$ is a form of degree $p$ and order less than $w$. Note that $h(\underline{x})$ belongs to $X_{w-1} X^{m-1}$. By hypothesis, $f(\underline{x})$ belongs to $X_{i-1} X^{m-1}$. But, since $w \geq i$, we have $X_{i-1} X^{m-1} \subseteq X_{w-1} X^{m-1}$. Thus $x_{w} f_{1}(\underline{x})=f(\underline{x})-h(\underline{x})$ belongs to 
$X_{w-1} X^{m-1}$. It follows that $f_{1}(\underline{x})$ belongs to $\left(X_{w-1} X^{m-1}: x_{w}\right) \cap X^{p-1}$ and hence also to $\left(X_{w-1} X^{m-1}: x_{w}\right) \cap X^{m}$, which by hypothesis equals $X_{w-1} X^{m-1}$. By the induction hypothesis on $p$, there exists a form $g_{1}$ in $S$ of degree $p-1$ and order less than $w$ such that $f_{1} \equiv g_{1} \bmod \mathbf{N}_{m}$.

Note that $f\left(=U_{w} f_{1}+h\right)$ is congruent to $U_{w} g_{1}+h$ modulo $\mathbf{N}_{m}$. Since $f_{1}(\underline{x})=g_{1}(\underline{x})$, it follows that $\left(U_{w} g_{1}+h\right)(\underline{x})$ is equal to $f(\underline{x})$ and hence belongs to $X_{i-1} X^{m-1}$. Now, since $U_{w} g_{1}+h$ has degree $p$ and order less than $w$, it follows from the induction hypothesis on $w$ that there exists a form $g$ of degree $p$ and order less than $i$ which is congruent modulo $\mathbf{N}_{m}$ to $U_{w} g_{1}+h$ (and hence also to $f$ ).

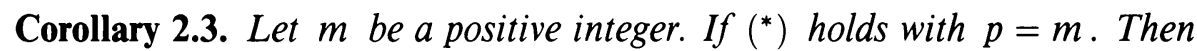

(1) $\left(^{*}\right)$ holds for every $p \geq m$, and

(2) the relation type of $X$ is at most $m$.

Proof. (1) Clearly $\left(X_{i-1} X^{p-1}: x_{i}\right) \cap X^{p} \supseteq X_{i-1} X^{p-1}$. To see the other containment, notice first that, for an element $a$ of $X^{p}$, there exists a form in $S$ of degree $p$ such that $a=f(\underline{x})$. If such an element $a$ also belongs to $\left(X_{i-1} X^{p-1}: x_{i}\right)$, then, for $p \geq m, f(\underline{x})=a \in\left(X_{i-1} X^{m-1}: x_{i}\right) \cap X^{m}$, which by hypothesis equals $X_{i-1} X^{m-1}$. Now, by the proposition, there exists a form $g$ is $S$ of degree $p$ and order less than $i$ that is congruent modulo $\mathbf{N}_{m}$ to $f$. In particular $f(\underline{x})=g(\underline{x}) \in X_{i-1} X^{p-1}$.

(2) Let $f$ be a relation of degree $p \geq m$ on $x_{1}, \ldots, x_{n}$. The proposition, applied with $i=1$, guarantees the existence of a form $g$ of degree $p$ and order less than 1 that is congruent modulo $\mathbf{N}_{m}$ to $f$. But then $g=0$, since 0 is the only form of order 0 .

Proposition 2.4. Let $x_{1}, \ldots, x_{n}$ be an ordered sequence of elements of a ring $R$, and let $m$ be a fixed positive integer. If $(\dagger)$ holds with $p=m$, then $\mathbf{N}_{m+1}=\mathbf{N}_{m}$, that is, every relation of degree $m+1$ on $x_{1}, \ldots, x_{n}$ is in the ideal generated by the relations of degree $m$.

Proof. Let $f$ be a relation of degree $m+1$. We use induction on the order of $f$ to show that $f$ belongs to $\mathbf{N}_{m}$. Let $w$ denote the order of $f$. If $w=0$, then $f=0$, and so the conclusion holds.

Now assume that $w \geq 1$ and write $f=U_{w} f_{1}+h$ where $f_{1}$ is a form of degree $m$ and $h$ is a form of degree $m+1$ and order less than $w$. Note that $x_{w} f_{1}(\underline{x})=\left(U_{w} f_{1}\right)(\underline{x})=f(\underline{x})-h(\underline{x})=-h(\underline{x}) \in X_{w-1} X^{m}$. Thus $f_{1}(\underline{x})$ belongs to $\left(X_{w-1} X^{m}: x_{w}\right) \cap X^{m}$, which by hypothesis equals $X_{w-1} X^{m-1}$. This means that there exists a form $g$ in $S$ of degree $m$ and order less than $w$ such that $f_{1}(\underline{x})=g(\underline{x})$. Thus $f_{1}-g$ is a relation, and since it has degree $m$, it belongs to $\mathbf{N}_{m}$. Now $f-U_{w}\left(f_{1}-g\right)=U_{w} g+h$ is also a relation of degree $m+1$. Since $U_{w} g+h$ has order less than $w$, it follows from the induction hypothesis that it (and hence also $f$ ) belongs to $\mathbf{N}_{m}$.

We now answer, in the negative, two questions of Costa [C] concerning what he has termed "sequences of linear type".

Definition 2.5 ([C]). An ordered sequence $x_{1}, \ldots, x_{n}$ of elements of a ring is called a sequence of linear type if $X_{i}$ is an ideal of linear type for every $i$, $1 \leq i \leq n$. 
Notice that $d$-sequences are sequences of linear type. But not every sequence of linear type is a $d$-sequence (see, for example, $\S 6$ ). Costa asks whether every ideal generated by a sequence of linear type is actually generated by a $d$ sequence. The following example shows that this is not true.

Example 2.6. Let $R=k\left[X_{i j}\right]_{1 \leq i, j \leq 3}$, where $k$ is a field and $X_{i j}$ are indeterminates. It can be checked, using "MACAULAY" for example, that the $2 \times 2$ minors of the $3 \times 3$ matrix $\left(X_{i j}\right)$ form a sequence of linear type in the following order: $-X_{12} X_{21}+X_{11} X_{22}, X_{12} X_{31}-X_{11} X_{32},-X_{22} X_{31}+X_{21} X_{32}$, $X_{13} X_{21}-X_{11} X_{23},-X_{13} X_{31}+X_{11} X_{33}, X_{23} X_{31}-X_{21} X_{33},-X_{13} X_{22}+X_{12} X_{23}$, $X_{13} X_{32}-X_{12} X_{33},-X_{23} X_{32}+X_{22} X_{33}$. (Do they form a sequence of linear type in any order?) The ideal generated by the $2 \times 2$ minors cannot, however, be generated by a $d$-sequence (see [Vas, Example 5.10]). Huneke [H4] has shown that the ideal generated by the $t \times t$ minors of a $(t+1) \times(t+1)$ generic matrix (over a domain) is of linear type.

The second question arises out of the following result in [C]: If $x_{1}, x_{2}$ is a sequence of linear type then so is $x_{1}^{s}, x_{2}^{s}$ for any positive integer $s$. Costa asks whether the same is true for sequences of linear type of arbitrary length. The $2 \times 2$ minors of a generic $2 \times 3$ matrix over a field provide a counterexample to this question even for $s=2$ (see Example 4.1); this also was checked using "MACAULAY".

\section{WEAK $d$-SEQUENCES GENERATE IDEALS OF QUADRATIC TYPE}

As the title of this section suggests, its purpose is to settle the following conjecture of Huneke in the affirmative : Ideals generated by weak $d$-sequences are of quadratic type (see Corollary 3.6). The proof of the Main Theorem 3.6 of this section suggests the definition of quadratic sequences, which are not only simpler to define and more general than weak $d$-sequences, but also, as we show in $\S 5$, the whole theory of weak $d$-sequences extends to these sequences. We begin by establishing notation.

By a poset, we mean a partially ordered set. Let $\Lambda$ be a finite poset. A subset $\Sigma$ of $\Lambda$ is said to be an ideal if it satisfies the following property :

$$
\text { if } \sigma \in \Sigma, \lambda \in \Lambda \text {, and } \lambda \leq \sigma \text {, then } \lambda \in \Sigma \text {. }
$$

Throughout this section, small Greek letters will be used exclusively to denote elements of a finite poset. Let $\left\{x_{\lambda} \mid \lambda \in \Lambda\right\}$ be a set of elements of a ring $R$ indexed by $\Lambda$. Given an ideal $\Sigma$ of $\Lambda$, we denote the ideal $\left(x_{\sigma} \mid \sigma \in \Sigma\right)$ of $R$ by $X_{\Sigma}$ and call it the $\Lambda$-ideal (of $R$ ) defined by $\Sigma$. An ideal of $R$ is called a $\Lambda$-ideal if it equals $X_{\Sigma}$ for some ideal $\Sigma$ of $\Lambda$. The empty subset of $\Lambda$ is an ideal and the $\Lambda$-ideal defined by it is the ideal 0 . We write $X$ instead of $X_{\Lambda}$; in other words, $X$ denotes the ideal $\left(x_{\lambda} \mid \lambda \in \Lambda\right)$ of $R$.

Given an ideal $\Sigma$ and an element $\lambda$ of $\Lambda$, we say that $\lambda$ lies just above $\Sigma$ if $\lambda \notin \Sigma$ but every element $\sigma$ of $\Lambda$ such that $\sigma \lessgtr \lambda$ belongs to $\Sigma$. We say that $\lambda$ lies inside or just above $\Sigma$ if it either belongs to $\Sigma$ or is just above $\Sigma$.

Definition 3.1 ([H2, Definition 1.1]). We say that $\left\{x_{\lambda} \mid \lambda \in \Lambda\right\} \subseteq R$ is a weak $d$ sequence (indexed by $\Lambda$ ) if, for every pair $(\Sigma, \lambda)$ where $\Sigma$ is an ideal of $\Lambda$ and $\lambda$ is an element of $\Lambda$ that lies just above (equivalently, lies inside or just above) 
$\Sigma$, the following conditions hold ( $\Theta$ denotes the subset $\left\{\theta \in \Lambda \mid x_{\theta} x_{\lambda} \in X_{\Sigma}\right\}$ of $\Lambda)$ :

(1) $\Theta$ is an ideal of $\Lambda$;

(2) $\left(X_{\Sigma}: x_{\lambda}\right) \cap X=X_{\Theta}$

(3) $x_{\lambda} X_{\boldsymbol{\theta}} \subseteq X_{\Sigma} X$; and

(4) either $\lambda \in \Theta$ or $\left(X_{\Sigma}: x_{\lambda}\right)=\left(X_{\Sigma}: x_{\lambda}^{2}\right)$.

Definition 3.2 ([H2, Definition 2.1]; see remark below). Let $I$ be an ideal of $R$. Suppose that $\left\{x_{\lambda} \mid \lambda \in \Lambda\right\} \subseteq R$ is such that $\left\{\bar{x}_{\lambda} \mid \lambda \in \Lambda\right\} \subseteq R / I$ is a weak $d$-sequence, where $\bar{x}_{\lambda}$ denotes the image of $x_{\lambda}$ in $R / I$. Let $\Sigma$ be an ideal of $\Lambda$ and $\lambda$ be an element of $\Lambda$ that lies (inside or) just above $\Sigma$. Let $\Theta=\left\{\theta \in \Lambda \mid \bar{x}_{\theta} \bar{x}_{\lambda} \in \bar{X}_{\Sigma}\right\}$. By axiom (3), we have $x_{\lambda} X_{\Theta} \subseteq X_{\Sigma} X+I$. If, in addition to $\left\{\bar{x}_{\lambda} \mid \lambda \in \Lambda\right\} \subseteq R / I$ being a weak $d$-sequence, the following stronger version of axiom (3) holds, then we say that $\left\{x_{\lambda} \mid \lambda \in \Lambda\right\} \subseteq R$ is a weak $d$-sequence with respect to $I$ :

(3a) $x_{\lambda} X_{\boldsymbol{\theta}} \subseteq\left(X_{\Sigma}+I\right) X$.

Remark. Huneke's terminology for what we have called "weak $d$-sequence with respect to the ideal $I$ " is "weak $d$-sequence modulo $I$ ".

Let $X$ denote the ideal generated by elements of a weak $d$-sequence with respect to an ideal $I$. Theorem 2.1 of [H2] says that $I \cap X^{d} \subseteq I X^{d-1}+I^{2} X^{d-2}$ for every integer $d \geq 2$. It is immediate from the following theorem (see Corollary 3.7 below) that this can be improved to $I \cap X^{d} \subseteq I X^{d-1}$. This improvement enables us to use "Transitivity of Depth" and obtain Corollary 5.5, a result which for $d$-sequences is due to Huneke [H1, Corollary 4.1]. Another immediate corollary of the following theorem is that weak $d$-sequences generate ideals of quadratic type (see Corollary 3.8 below). While the theorem is applicable to weak $d$-sequences, the proof does not use the full strength of all axioms; it works with the less stringent condition of the following definition.

Definition 3.3. Let $I$ be an ideal of $R$, and use " -" to denote images in $R / I$. We say that $\left\{x_{\lambda} \mid \lambda \in \Lambda\right\} \subseteq R$ is a quadratic sequence with respect to the ideal $I$ if, for every pair $(\Sigma, \lambda)$ where $\Sigma$ is an ideal of $\Lambda$ and $\lambda$ is an element of $\Lambda$ that lies (inside or) just above $\Sigma$, there exists an ideal $\Theta$ of $\Lambda$ such that

(1) $\left(\bar{X}_{\Sigma}: \bar{x}_{\lambda}\right) \cap \bar{X} \subseteq \bar{X}_{\boldsymbol{\Theta}}$, and

(2) $x_{\lambda} X_{\Theta} \subseteq\left(X_{\Sigma}+I\right) X$.

A quadratic sequence with respect to the ideal 0 is called, simply, a quadratic sequence. Note that, in view of (2), we would get an equivalent definition if the containment relation in (1) were replaced by an equality. Motivation for the nomenclature "quadratic sequence" is provided by Corollary 3.8 below. Condition (1) can be rewritten as follows:

$\left(1^{\prime}\right) \quad\left(X_{\Sigma}+I: x_{\lambda}\right) \cap X \subseteq X_{\Theta}+I$.

For an example of a quadratic sequence that is not a weak $d$-sequence, let

$$
R=\frac{k[X, Y, Z, W]}{(X Y, X W-Y Z)}
$$

where $k$ is a field. The images of $X$ and $Y$ in $R$ form a quadratic sequence on the discrete poset of two elements. But they are not a weak $d$-sequence since the image $\bar{W}$ of $W$ belongs to $\left(0: \bar{X}^{2}\right) \backslash(0: \bar{X})$. 
The basic facts about quadratic sequences contained in the following remark are used at various points in the sequel. The proofs are routine verifications.

Remark 3.4. A weak $d$-sequence with respect to an ideal is clearly a quadratic sequence with respect to that ideal. A quadratic sequence with respect to an ideal $I$ is also a quadratic sequence with respect to $I+X_{\Sigma}$ for any ideal $\Sigma$ of the indexing poset. In particular, a quadratic sequence indexed by $\Lambda$ is a quadratic sequence with respect to any $\Lambda$-ideal. If $\left\{x_{\lambda} \mid \lambda \in \Lambda\right\} \subseteq R$ is a quadratic sequence and $\Sigma$ is an ideal of $\Lambda$, then $\left\{\bar{x}_{\lambda} \mid \lambda \in \Lambda \backslash \Sigma\right\} \subseteq R / X_{\Sigma}$ is a quadratic sequence, where "-" is used to denote images modulo $X_{\Sigma}$.

In what follows, $S$ denotes the graded polynomial ring $R\left[U_{\lambda} \mid \lambda \in \Lambda\right]$ over $R$ in as many indeterminates as there are elements of $\Lambda$. Elements of $R$ have degree 0 and each $U_{\lambda}$ has degree 1 . For $f \in S$, let $f(\underline{x})$ denote the image of $f$ under the evaluation map from $S$ to $R$ defined by $U_{\lambda} \mapsto x_{\lambda}$.

Definition 3.5. If $f$ is a form in $S$ and $\Sigma$ is an ideal of $\Lambda$, we say that $\Sigma$ divides $f$ and write $\Sigma \mid f$ if every (monomial) term of $f$ is divisible by one of the indeterminates $U_{\sigma}, \sigma \in \Sigma$; it is not required that a fixed indeterminate $U_{\sigma}$ divide every term. Note that $\Sigma \mid f$ if and only if $f$ belongs to the ideal $\left(U_{\sigma} \mid \sigma \in \Sigma\right)$ of $S$.

If $\Sigma \mid f$, and $g$ is any form in $S$, then $\Sigma \mid f g$. If $\Sigma \mid f$ and $\Sigma \mid g$, then $\Sigma \mid f+g$. The form 0 is divisible by every ideal of $\Lambda$. The empty subset of $\Lambda$ divides only the form 0 .

Theorem 3.6. Let $\Lambda$ be a finite poset, let $R$ be a ring, and let $I$ be an ideal of $R$. Let $\left\{x_{\lambda} \mid \lambda \in \Lambda\right\} \subseteq R$ be a quadratic sequence with respect to I. Let $\mathbf{N}_{3 / 2}$ be the ideal of the polynomial ring $S=R\left[U_{\lambda} \mid \lambda \in R\right]$ generated by quadratic forms $q$ such that $q(\underline{x})$ belongs to $I X$. (The following remark explains the choice of the subscript 3/2.) Let $\Sigma$ be an ideal of $\Lambda$ and $f$ be a form of degree $d \geq 2$ in $S$ such that $\overline{f(\underline{x})}$ belongs to $\bar{X}_{\Sigma}$, where "- " is used to denote images in $R / I$. Then there exists a form $g$ in $S$ of degree $d$ such that $f \equiv g \bmod \mathbf{N}_{3 / 2}$ and $\Sigma$ divides $g$.

Remark. Given a positive integer $d$, it is natural to denote by $\mathbf{N}_{d}$ the ideal generated by forms of degree $d$ that yield an element of $I$ when evaluated at $\underline{x}$. Notice that $\mathbf{N}_{1} \subseteq \mathbf{N}_{3 / 2} \subseteq \mathbf{N}_{2}$, which provides the rationale for the notation " $\mathbf{N}_{3 / 2}$ ". It follows from the theorem (see Corollary 3.7 below) that, for an element $f$ of $\mathbf{N}_{d}$, we have $f(\underline{x}) \in I X^{d-1}$. In particular, $\mathbf{N}_{3 / 2}=\mathbf{N}_{2}$.

Proof of the theorem. We use a double induction, the first on $d$, next on the number of elements of $\Lambda \backslash \Sigma$. If $\Sigma=\Lambda$, then the conclusion is obvious (take $g=f)$. Assume that $\Sigma \subsetneq \Lambda$. Let $\lambda$ be an element of $\Lambda$ that is just above $\Sigma$. Note that $\overline{f(\underline{x})}$ belongs to $\bar{X}_{\Sigma \cup\{\lambda\}}$. Since the number of elements in $\Lambda \backslash(\Sigma \cup\{\lambda\})$ is strictly less than that of $\Lambda \backslash \Sigma$, it follows from the induction hypothesis that there exists a form $g^{\prime}$ in $S$ of degree $d$ such that $f \equiv g^{\prime} \bmod \mathbf{N}_{3 / 2}$ and $\Sigma \cup\{\lambda\}$ divides $g^{\prime}$. Thus, we may, after a change of notation (writing $f$ instead of $g^{\prime}$ ), assume that $f=U_{\lambda} f^{\prime}+h$, where $f^{\prime}$ and $h$ are forms of degrees $d-1$ and $d$ respectively and $\Sigma \mid h$. Further, we may subtract $h$ from $f$ without affecting the hypothesis or the conclusion and assume that $f=U_{\lambda} f^{\prime}$.

Let $\Theta$ be an ideal of $\Lambda$ such that conditions (1) and (2) of Definition 3.3 are satisfied. Note that $\overline{f^{\prime}(\underline{x})}$ belongs to $\left(\bar{X}_{\Sigma}: \bar{x}_{\lambda}\right) \cap \bar{X}$ and hence also to $\bar{X}_{\boldsymbol{\theta}}$. By 
the induction hypothesis on the degree, there exists a form $g^{\prime}$ in $S$ of degree $d-1$ such that $f^{\prime} \equiv g^{\prime} \bmod \mathbf{N}_{3 / 2}$ and $\Theta \mid g^{\prime}$. (In the base case $d=2, f^{\prime}$ is linear; so there exists a linear form $g^{\prime}$ such that $\Theta \mid g^{\prime}$ and $\left(f^{\prime}-g^{\prime}\right)(\underline{x})$ belongs to $I$.) Thus $U_{\lambda} f^{\prime} \equiv U_{\lambda} g^{\prime} \bmod \mathbf{N}_{3 / 2}$ (even in the base case $\left.d=2\right)$. Subtracting $U_{\lambda}\left(f^{\prime}-g^{\prime}\right)$ from $f$, we may assume that $f=U_{\lambda} g^{\prime}$.

Write $g^{\prime}=\sum_{\theta \in \Theta} U_{\theta} g_{\theta}^{\prime}$. For each $\theta \in \Theta$, there exists, by condition (2) of Definition 3.3, a quadratic form $q_{\theta}$ such that $\Sigma \mid q_{\theta}$ and $\left(U_{\lambda} U_{\theta}-q_{\theta}\right)(\underline{x})$ belongs to $I X$. Let $g=\sum_{\theta \in \Theta} q_{\theta} g_{\theta}^{\prime}$. Since $\Sigma$ divides each $q_{\theta}$, it also divides $g$. Since each $U_{\lambda} U_{\theta}-q_{\theta}$ is (by definition) a generator of $\mathbf{N}_{3 / 2}$, it follows that $f-g=\sum_{\theta \in \boldsymbol{\Theta}}\left(U_{\lambda} U_{\theta}-q_{\theta}\right) g_{\theta}^{\prime}$ belongs to $\mathbf{N}_{3 / 2}$.

Huneke proved a slightly weaker version of the following corollary for weak $d$-sequences [H2, Theorem 2.1] (see discussion preceding Definition 3.3).

Corollary 3.7. If $\left\{x_{\lambda} \mid \lambda \in \Lambda\right\} \subseteq R$ is a quadratic sequence with respect to an ideal $I$, then $I \cap X^{d} \subseteq I X^{d-1}$ for every integer $d \geq 1$. In particular, if $\left\{x_{\lambda} \mid \lambda \in\right.$ $\Lambda\} \subseteq R$ is a quadratic sequence, then $X_{\Sigma} \cap X^{d}=X_{\Sigma} X^{d-1}$ for any ideal $\Sigma$ of $\Lambda$.

Proof. To prove the first statement, notice first that it is obvious for $d=1$. Now let $d \geq 2$. For $a \in I \cap X^{d}$, there exists a form $f$ in $S$ of degree $d$ such that $f(\underline{x})=a$. Let $\Sigma$ be the empty subset of $\Lambda$ and apply the theorem. Conclude that $f$ belongs to $\mathbf{N}_{3 / 2}$. Write $f=\sum_{i} q_{i} f_{i}$, where each $q_{i}$ is a quadratic form such that $q_{i}(\underline{x}) \in I X$, and each $f_{i}$ is a form of degree $d-2$. For each $i,\left(q_{i} f_{i}\right)(\underline{x})=q_{i}(\underline{x}) f_{i}(\underline{x})$ belongs to $I X \cdot X^{d-2}=I X^{d-1}$. Hence so does $f(\underline{x})=a$.

To prove the second statement, it suffices to show that $X_{\Sigma} \cap X^{d} \subseteq X_{\Sigma} X^{d-1}$ because the other containment is clear. A routine verification shows that every quadratic sequence indexed by $\Lambda$ is a quadratic sequence with respect to any $\Lambda$-ideal (see Remark 3.4). Now set $I=X_{\Sigma}$ in the first statement.

Remark. In fact, we have $I \cap X^{d}=\left(I X \cap X^{2}\right) X^{d-2}$ for $d \geq 2$ in the above corollary.

Proof. In the proof above, notice that $q_{i}(\underline{x}) \in I X \cap X^{2}$ and that $f_{i}(\underline{x}) \in$ $X^{d-2}$.

Corollary 3.8. If $\left\{x_{\lambda} \mid \lambda \in \Lambda\right\} \subseteq R$ is a quadratic sequence, then $X$ is of quadratic type.

Proof. Let $f$ be a form of degree $d \geq 2$ such that $f(\underline{x})=0$. Apply the theorem with $I=0$ and $\Sigma$ equal to the empty subset of $\Lambda$ to conclude that $f$ belongs to $\mathbf{N}_{3 / 2}$. But, since $I=0$, it follows from the definition of $\mathbf{N}_{3 / 2}$ that it is the ideal generated by quadratic forms $q$ such that $q(\underline{x})=0$.

Corollary 3.7 gives examples of Ratliff-Rush ideals, as follows. Recall the following definition.

Definition 3.9 [HLS]. A regular ideal $I$ (that is, one which contains a nonzerodivisor) of a ring $R$ is called Ratliff-Rush if $I=\bigcup_{n}\left(I^{n+1}: I^{n}\right)$.

Proposition 3.10. If $a$ is a nonzero divisor of a ring $R$ and $X$ is an ideal of $R$ such that $(a) \cap X^{d}=a X^{d-1}$ for all $d \geq 1$, then the ideal $(a)+X$ is Ratliff-Rush. Proof. Let $J$ denote the ideal $(a)+X$. We claim that $\left(J^{d+1}: a^{d}\right)=J$, which shows in particular that $\left(J^{d+1}: J^{d}\right)=J$. We prove the claim by induction on 
$d$. For $d=0$, the claim is clear. Let $z \in\left(J^{d+1}: a^{d}\right)$. Write $z a^{d}=a y+b$, where $y \in J^{d}$ and $b \in X^{d+1}$. Now, $b \in(a) \cap X^{d+1}=a X^{d}$. Write $b=a b^{\prime}$, where $b^{\prime} \in X^{d}$. Substituting this into $z a^{d}=a y+b$, and cancelling $a$, we get $z a^{d-1}=y+b^{\prime} \in J^{d}$. Thus $z$ belongs to $\left(J^{d}: a^{d-1}\right)$, which equals $J$ by the induction hypothesis.

Corollary 3.11. (i) In a domain, quadratic sequences generate Ratliff-Rush ideals.

(2) Let a be a nonzero divisor of a ring $R$. Let $X$ be an ideal of $R$ such that the ideal $\bar{X}$ of the ring $R /(a)$ is of linear type. Then the ideal $(a)+X$ is Ratliff-Rush.

Proof. (1) Let $R$ be a domain and $\left\{x_{\lambda} \mid \lambda \in \Lambda\right\} \subseteq R$ be a quadratic sequence. Choose a minimal element $\alpha$ of the indexing poset $\Lambda$. Notice that $\left\{x_{\lambda} \mid \lambda \in\right.$ $\Lambda, \lambda \neq \alpha\}$ is a quadratic sequence with respect to the ideal $\left(x_{\alpha}\right)$. The result now follows from the above proposition and the first statement of Corollary 3.7.

(2) A routine verification shows that since $\bar{X}$ is an ideal of linear type, (a) $\cap X^{d+1}=a X^{d}$.

\section{EXAMPLES}

In this section, we give examples of quadratic sequences. Other than recalling examples of $d$-sequences and weak $d$-sequences from the literature, we show that ideals of small analytic deviation studied recently by Huckaba and Huneke [HuH1, HuH2] are generated by weak $d$-sequences. The desire to prove that these ideals were of quadratic type was the starting point of the work of this paper. We first recall some examples of $d$-sequences. The following list is by no means exhaustive. For more examples of $d$-sequences, see [Fi; H1; H3; HSV1, $\S 5$; SV; SV1].

Example 4.1 (The maximal minors of a generic $n \times(n+1)$ matrix). Let $n$ be a positive integer, and let $R=k\left[X_{i j} \mid 1 \leq i \leq n, 1 \leq j \leq n+1\right]$, where $k$ is a field and $X_{i j}$ are indeterminates. The maximal minors of the $n \times(n+1)$ matrix $\left(X_{i j}\right)$ form a $d$-sequence in any order.

Example 4.2 ([HU, Proposition 6.1]). If $I$ is a licci ideal of a formal power series ring $R$ over a field, and if $R / I$ is rigid, then $I$ is generated by a $d$ sequence.

Example 4.3 ([H6, Theorem 2.4]). Let $R$ be a Cohen-Macaulay local ring. Let $I$ be a strongly Cohen-Macaulay ideal of $R$ such that $\mu\left(I_{\mathbf{p}}\right) \leq \mathrm{ht}(\mathbf{p}) \quad \forall \mathbf{p} \in$ $\operatorname{Spec}(R), \mathbf{p} \supseteq I$. Then $I$ is generated by a Cohen-Macaulay $d$-sequence (a $d$ sequence is said to be Cohen-Macaulay if all its related ideals (see Definition 5.3) are Cohen-Macaulay.)

Next we give examples of weak $d$-sequences. It is easy to see that $d$ sequences are weak $d$-sequences. Straightening-closed ideals of graded ordinal Hodge algebras are the other major class of weak $d$-sequences. The theory of Hodge algebras was developed by DeConcini, Eisenbud, and Procesi in [DEP]. Ordinal hodge algebras are also called algebras with straightening law. For the definition of a straightening-closed ideal we refer the reader to [BST]. For examples and more information, see [BST; BV, Chapters 4 and 5; DEP; H2; H5]. 
Proposition 4.4 [H2]. Elements of an ordinal Hodge algebra indexed by a straightening-closed ideal of the underlying poset form a weak $d$-sequence (and therefore also a quadratic sequence).

We now show that certain ideals of small analytic deviation are generated by weak $d$-sequences. Let $I$ be an ideal of a Noetherian local ring $R$. The analytic deviation of $I$ is $\operatorname{ad}(I):=l(I)-\mathrm{ht}(I)$, where $l(I)$ is the analytic spread of $I$ (see Definition 7.1) and $\mathrm{ht}(I)$ is the height of $I$. Huckaba and Huneke have recently studied ideals of small analytic deviation [HuH1, HuH2]. Recall that an ideal $J \subseteq I$ is called a reduction of $I$ if $J I^{n}=I^{n+1}$ for some non-negative integer $n$ (see [NR]). A reduction is called a minimal reduction if there does not exist a reduction strictly contained in $J$. A basic fact is that the number of generators of any minimal reduction equals the analytic spread of $I$ (see [NR]). Given a reduction $J$ of $I$, the reduction number of $I$ with respect to $J$ is defined by $r_{J}(I)=\min \left\{n \in \mathbb{Z}_{+} \cup\{0\} \mid J I^{n}=I^{n+1}\right\}$. The reduction number of $I$ is defined by $r(I)=\min \left\{r_{J}(I) \mid J\right.$ is a minimal reduction of $\left.I\right\}$. The hypothesis in Theorem 4.5 below (respectively Theorem 4.9 ) are the same as in Theorem 2.2 of [HuH1] (respectively Theorem 3.1 of [HuH1]). For examples of ideals to which these theorems can be applied, see $\S 4$ of [HuH1]; see also Corollary 4.10 .

Theorem 4.5. Let $R$ be a Cohen-Macaulay local ring and $I$ an ideal of $R$ of analytic deviation 1 and height $d \geq 1$. Assume that the minimal primes of $I$ all have the same height and associated primes of I have height at most $d+1$. Assume also that $I$ is generically a complete intersection (i.e., $I_{\mathbf{p}}$ is generated by a regular sequence in $R_{\mathbf{p}}$ for every minimal prime $\mathbf{p}$ over $\left.I\right)$ and that there exists a minimal reduction $J$ of $I$ such that $r_{J}\left(I_{Q}\right) \leq 1$ for every prime ideal $Q \supseteq I$ with $\operatorname{ht}(Q / I)=1$. Then $I$ is generated by a weak $d$-sequence.

Before proving the theorem we introduce the following definition.

Definition 4.6. An ordered sequence $x_{1}, \ldots, x_{n}$ of elements of a ring $R$ is said to be a $d$-sequence with respect to an ideal $I \supseteq\left(x_{1}, \ldots, x_{n}\right)$, if

$$
\left(X_{i-1}: x_{i}\right) \cap I=X_{i-1} \quad \forall 1 \leq i \leq n .
$$

Compare this with condition (2) of the definition of a $d$-sequence in the second paragraph of $\S 1$.

Proposition 4.7. Let $x_{1}, \ldots, x_{n}$ be a $d$-sequence with respect to a finitely generated ideal $I$ of an arbitrary commutative ring $R$. Assume that $\left(x_{1}, \ldots, x_{n}\right) I=$ $I^{2}$ and that $x_{i} \notin\left(x_{1}, \ldots, \hat{x}_{i}, \ldots, x_{n}\right) \quad \forall 1 \leq i \leq n$. Then $I$ is generated by $a$ weak $d$-sequence (and therefore also a quadratic sequence).

Proof. Choose elements $y_{1}, \ldots, y_{m}$ in $I$ such that they form an unshortenable generating set for the ideal $I$ modulo $X=\left(x_{1}, \ldots, x_{n}\right)$. Let $\Lambda$ be a poset with $n+m$ elements $\alpha_{1}, \ldots, \alpha_{n}, \beta_{1}, \ldots, \beta_{m}$ in the following order : $\alpha_{1}<$ $\ldots<\alpha_{n} ; \alpha_{i}<\beta_{j} \forall 1 \leq i \leq n, 1 \leq j \leq m$. Let $x_{i}$ be indexed by $\alpha_{i}$ and $y_{j}$ by $\beta_{j}$. We claim that $x_{1}, \ldots, x_{n}, y_{1}, \ldots, y_{m}$ form a weak $d$-sequence in this order.

To prove the claim, we adopt the notation of Definition 3.1. The following two situations have to be checked :

(1) $\Sigma=\left\{\alpha_{1}, \ldots, \alpha_{i}\right\}$ where $1 \leq i \leq n-1$ and $\lambda=\alpha_{i+1}$. 
(2) $\Sigma=\left\{\alpha_{1}, \ldots, \alpha_{n}\right\} \cup \Omega$ where $\Omega$ is some subset of $\left\{\beta_{1}, \ldots, \beta_{m}\right\}$ and $\lambda=\beta_{j}$ such that $\beta_{j} \notin \Omega$.

In the first case we claim that $\Theta=\Sigma=\left\{\alpha_{1}, \ldots, \alpha_{i}\right\}$. This follows from the assumption that $\left(X_{\Sigma}: x_{\lambda}\right) \cap I=X_{\Sigma}$ and our minimality hypotheses which force $y_{j} \notin X_{\Sigma} \quad \forall 1 \leq j \leq m$ and $x_{j} \notin X_{\Sigma}$ if $i<j \leq n$. The first three axioms of Definition 3.1 are clearly satisfied. For the last, suppose that $a x_{i+1}^{2} \in X_{\Sigma}=$ $\left(x_{1}, \ldots, x_{i}\right)$. Then $a x_{i+1} \in\left(X_{\Sigma}: x_{\lambda}\right) \cap I=X_{\Sigma}$, which implies $a \in\left(X_{\Sigma}: x_{\lambda}\right)$.

In the second case $\Theta=\Lambda$, and all axioms are easily checked. To verify the third axiom, note $\left(x_{1}, \ldots, x_{n}\right) I=I^{2}$.

Proof of Theorem 4.5. By Theorem 2.2 of [HuH1], $r_{J}(I) \leq 1$. Since $I$ is generically a complete intersection and $l(I)=\mathrm{ht}(I)+1$, we may assume (using a general position argument; see [HuH1]) that $J=\left(x_{1}, \ldots, x_{d}, c\right)$ where $x_{1}, \ldots, x_{d}$ is a regular sequence that generates $I$ generically. We claim that $x_{1}, \ldots, x_{d}, c$ is a $d$-sequence with respect to $I$. Once the claim is proved, we are done by using the above proposition.

To prove the claim, we need only check that $\left(\left(x_{1}, \ldots, x_{d}\right): c\right) \cap I=$ $\left(x_{1}, \ldots, x_{d}\right)$, since $x_{1}, \ldots, x_{d}$ is a regular sequence. It is enough to check this locally at every associated prime of $\left(x_{1}, \ldots, x_{d}\right)$. The associated primes of $\left(x_{1}, \ldots, x_{d}\right)$ are its minimal primes. If $Q$ is a minimal prime of $\left(x_{1}, \ldots, x_{d}\right)$ that does not contain $c$, then $\left(\left(x_{1}, \ldots, x_{d}\right): c\right)_{Q}=\left(x_{1}, \ldots, x_{d}\right)_{Q}$ so equality holds. If $Q$ contains $c$, then $\left(x_{1}, \ldots, x_{d}\right)_{Q}=I_{Q}$ because $x_{1}, \ldots, x_{d}$ generate $I$ generically, so equality holds again.

Corollary 4.8 [HuH1, Theorem 4.5; MS, Theorem 4.1]. Let I be as in the theorem. Then $I$ is of quadratic type.

Proof. Combine the above theorem with Corollary 3.8 .

Theorem 4.9. Let I be a Cohen-Macaulay ideal of analytic deviation 2 of a Gorenstein local ring. Assume that $I$ has height $d \geq 2, I_{Q}$ is a complete intersection for all primes $Q \supseteq I$ such that $\mathrm{ht}(Q / I)=1$, and there exists $a$ minimal reduction $K$ of $I$ such that $r_{K}\left(I_{Q}\right) \leq 1$ for every prime ideal $Q \supseteq I$ with $\mathrm{ht}(Q / I)=2$. Then $I$ is generated by a weak $d$-sequence. In particular, I is of quadratic type.

Proof. By [HuH1, Theorem 3.1], $r_{K}(I) \leq 1$, that is, $K I=I^{2}$. By [HuH2, Lemma 3.5], there exists a minimal generating set $\left\{a_{1}, \ldots, a_{d}, x, y\right\}$ of $K$ satisfying

(1) $a_{1}, \ldots, a_{d}$ is a regular sequence;

(2) $\left(\left(a_{1}, \ldots, a_{d}\right): x\right) \cap I=\left(a_{1}, \ldots, a_{d}\right)$; and

(3) $\left(\left(a_{1}, \ldots, a_{d}, x\right): y\right) \cap I=\left(a_{1}, \ldots, a_{d}, x\right)$.

In other words, $K$ is generated by a $d$-sequence with respect to the ideal $I$. Apply Proposition 4.7.

Corollary 4.10 (See Corollary 3.34 of [HuH2]). Let $R$ be a regular local ring and $P$ be a Cohen-Macaulay prime ideal of $R$ of dimension 3. If powers of $P$ coincide with its symbolic powers, then $P$ is of quadratic type.

Proof. From Burch's inequality (see $\S 7), P^{n}=P^{(n)}$ for $n \geq 1$ implies that $\operatorname{ad}(P) \leq 2$. If $Q$ is a prime containing $P$ such that $\operatorname{ht}(Q / P)=1$, then $P_{Q}$ 
is a complete intersection, since powers are symbolic powers. If $\operatorname{ad}(P)=0$, then $P$ is a complete intersection by [CN], hence of linear type. If $\operatorname{ad}(P)=1$, then the hypothesis of Theorem 4.5 are satisfied, hence $P$ is of quadratic type. Suppose that $\operatorname{ad}(P)=2$. For $Q$ a prime containing $P$ such that $\operatorname{ht}(Q / P) \leq 2$, Burch's inequality implies that $\operatorname{ad}\left(P_{Q}\right) \leq 1$. Applying Theorem 2.2 of [HuH1] to the ring $R_{Q}$, we see that there exists a minimal reduction $K$ of $P$ such that $r_{K}\left(P_{Q}\right) \leq 1$. Apply Theorem 4.9 to finish.

\section{Powers of IDEAls Generated by QUAdRatic SEQUeNCES}

In this section we extend the theory of $d$-sequences and weak $d$-sequences to quadratic sequences. The main result of this section is Theorem 5.4, which for weak $d$-sequences was proved by Huneke [H2, Theorem 2.2]. Our proof is more general yet simpler than Huneke's original proof. The result says that we can filter $R / X^{n}$, where $X$ is an ideal generated by elements of a quadratic sequence, such that the quotients are cyclic modules whose annihilators are "related ideals" of the quadratic sequence. Once this is established, the corollaries follow easily. Corollary 5.5 was proved by Huneke for $d$-sequences [ $\mathrm{H} 1$, Corollary 4.1], but it was not known for weak $d$-sequences. Corollary 3.7 enables us to prove it for quadratic sequences.

Lemma 5.1. If $\left\{x_{\lambda} \mid \lambda \in \Lambda\right\}$ is a quadratic sequence with respect to an ideal I, and $J$ is an ideal such that $J \cap X \subseteq I \subseteq J$, then $\left\{x_{\lambda} \mid \lambda \in \Lambda\right\}$ is a quadratic sequence with respect to $J$ as well.

Proof. For an ideal $\Sigma$ of $\Lambda$ and an element $\lambda$ of $\Lambda$ that is inside or just above $\Sigma$, there exists (by Definition 3.3 of a quadratic sequence with respect to an ideal) an ideal $\Theta$ of $\Lambda$ such that

(1) $\left(\bar{X}_{\Sigma}: \bar{x}_{\lambda}\right) \cap \bar{X} \subseteq \bar{X}_{\boldsymbol{\theta}}$, and

(2) $x_{\lambda} X_{\Theta} \subseteq\left(X_{\Sigma}+I\right) X$,

where " -" denotes images modulo $I$. Pulling (1) back to $R$, we get

$$
\left(\left(X_{\Sigma}+I\right): x_{\lambda}\right) \cap(X+I) \subseteq X_{\Theta}+I .
$$

It suffices to show that $\Theta$ satisfies the conditions

(i) $\left(X_{\Sigma}^{\prime}: x_{\lambda}^{\prime}\right) \cap X^{\prime} \subseteq X_{\Theta}^{\prime}$, and

(ii) $x_{\lambda} X_{\Theta} \subseteq\left(X_{\Sigma}+J\right) X$,

where "'" is used to denote images modulo $J$. Clearly (2) $\Rightarrow$ (ii), since $J \supseteq I$ by our hypothesis. Condition (i), when pulled back to $R$, yields the equivalent condition $\left(\left(X_{\Sigma}+J\right): x_{\lambda}\right) \cap(X+J) \subseteq X_{\boldsymbol{\theta}}+J$. This equivalent form of (i) is an easy consequence of (1) and the hypothesis that $J \cap X \subseteq I \subseteq J$, as we now show. Let $a \in X$ and $b \in J$ such that $(a+b) x_{\lambda}=c+d$ with $c \in X_{\Sigma}$ and $d \in J$. Then $d=(a+b) x_{\lambda}-c$ belongs to $J \cap X$ and hence also to $I$. Note also that $b x_{\lambda}$ belongs to $J X \subseteq J \cap X \subseteq I$. Thus $a x_{\lambda}=c+\left(d-b x_{\lambda}\right)$ belongs to $X_{\Sigma}+I$. This means that $a \in\left(\left(X_{\Sigma}+I\right): x_{\lambda}\right)$. Since $a \in X$ to begin with, it follows from (1) that $a \in X_{\boldsymbol{\theta}}+I$, which implies that $a+b$ belongs to $X_{\boldsymbol{\theta}}+I+J=X_{\boldsymbol{\theta}}+J$.

Corollary 5.2. If $\left\{x_{\lambda} \mid \lambda \in \Lambda\right\}$ is a quadratic sequence and $\alpha$ is a minimal element of $\Lambda$, then $\left\{x_{\lambda} \mid \lambda \in \Lambda\right\}$ is a quadratic sequence with respect to $\left(0: x_{\alpha}\right)$. 
Proof. Let $\Omega$ be an ideal of $\Lambda$ such that $\left(0: x_{\alpha}\right) \cap X \subseteq X_{\Omega}$ and $x_{\alpha} X_{\Omega}=0$ (to see that such an ideal exists, set $\Sigma$ equal to the empty subset of $\Lambda$ and $\lambda=\alpha$ in Definition 3.3 of a quadratic sequence). Note that, since $x_{\alpha} X_{\Omega}=0$, we actually have the equality $\left(0: x_{\alpha}\right) \cap X=X_{\Omega}$. As a routine check shows, every quadratic sequence indexed by $\Lambda$ is a quadratic sequence with respect to any $\Lambda$-ideal (see Remark 3.4). In particular, $\left\{x_{\lambda} \mid \lambda \in \Lambda\right\}$ is a quadratic sequence with respect to $X_{\Omega}$. Apply the lemma with $I=X_{\Omega}$ and $J=\left(0: x_{\alpha}\right)$.

Definition 5.3. Given a quadratic sequence $\left\{x_{\lambda} \mid \lambda \in \Lambda\right\}$, consider the ideals $\left(X_{\Sigma}: x_{\lambda}\right)+X$ obtained by letting $\Sigma$ vary over the set of all ideals of $\Lambda$ and $\lambda$ over all elements of $\Lambda$ that are inside or just above $\Sigma$. An ideal of $R$ is said to be related to the quadratic sequence if it is equal either to $X$ or to one of these ideals.

Theorem 5.4. Let $\Lambda$ be a finite poset, $R$ be a ring, and $\left\{x_{\lambda} \mid \lambda \in \Lambda\right\}$ be a quadratic sequence. Let $X$ denote the ideal $\left(x_{\lambda} \mid \lambda \in \Lambda\right)$. For every integer $n \geq 1$, there exists a filtration $R / X^{n}=M_{0} \supseteq M_{1} \supseteq \cdots \supseteq M_{k}=0$ of the $R$ module $R / X^{n}$ such that for every integer $i, 0 \leq i \leq k-1$, there exists an ideal $Y_{i}$ related to the quadratic sequence satisfying $M_{i} / M_{i+1} \cong R / Y_{i}$.

Proof. The proof proceeds by two inductions, the first on the number of elements of $\Lambda$, and the second on $n$. The base case $n=1$ of the second induction is clear since $X$ itself is a related ideal. The following proof of the induction step includes the proof of the base case of the first induction, namely, the case when $\Lambda$ is a singleton.

Fix a minimal element $\alpha$ of $\Lambda$ and write $x$ instead of $x_{\alpha}$. From the filtration $R / X^{n} \supseteq\left(x, X^{n}\right) / X^{n} \supseteq 0$, and the claim (proved below) that $\left(x, X^{n}\right) / X^{n}$ $\cong R /\left((0: x), X^{n-1}\right)$, it clearly suffices to show that $R /\left(x, X^{n}\right)$ and $R /\left((0: x), X^{n-1}\right)$ have filtrations of the desired form. To see the claim, consider the following display (clarifications follow):

$$
\frac{(x)+X^{n}}{X^{n}} \cong \frac{(x)}{X^{n} \cap(x)}=\frac{(x)}{x X^{n-1}} \cong \frac{R}{\left(x X^{n-1}: x\right)}=\frac{R}{(0: x)+X^{n-1}} .
$$

The first isomorphism follows from the general isomorphism $(A+B) / B$ $\cong A /(A \cap B)$ of submodules. The second isomorphism is also clear, since $\left(x X^{n-1}: x\right)$ is the kernel of the mapping from $R$ onto $(x) / x X^{n-1}$ defined by $1 \mapsto \bar{x}$. The last equality is a consequence of the general identity $(x \mathbf{A}: x)=$ $(0: x)+\mathbf{A}$ for an ideal $\mathbf{A}$ and an element $x$ of a ring. To see the first equality, set $\Sigma=\{\alpha\}$ (so that $X_{\Sigma}=(x)$ ) in the second statement of Corollary 3.7 to obtain $(x) \cap X^{n}=x X^{n-1}$. This finishes the proof of the claim.

To see that $R /\left(x, X^{n}\right)$ has a filtration of the desired form, let $\Lambda^{\prime}=\Lambda \backslash\{\alpha\}$. A routine verification shows that $\left\{\bar{x}_{\lambda} \mid \lambda \in \Lambda^{\prime}\right\} \subseteq R /(x)$ is a quadratic sequence, where " -" is used to denote images modulo $(x)$ (see Remark 3.4). Let $X^{\prime}$ denote the ideal $\left(x_{\lambda} \mid \lambda \in \Lambda^{\prime}\right)$. Since $\Lambda^{\prime}$ has fewer elements than $\Lambda$, it follows from our second induction that $\bar{R} /{\overline{X^{\prime}}}^{n}$ has a filtration in which the quotients are $\bar{R}$ modulo related ideals of $\left\{\bar{x}_{\lambda} \mid \lambda \in \Lambda^{\prime}\right\}$. But $\bar{R} /{\overline{X^{\prime}}}^{n} \cong R /\left(x, X^{\prime n}\right)=$ $R /\left(x, X^{n}\right)$, and the preimage in $R$ of a related ideal of $\left\{\bar{x}_{\lambda} \mid \lambda \in \Lambda^{\prime}\right\}$ is, as can be easily checked, a related ideal of $\left\{x_{\lambda} \mid \lambda \in \Lambda\right\}$. This proves that $R /\left(x, X^{n}\right)$ has a filtration of the required form. 
To see that $R /\left((0: x), X^{n-1}\right)$ has a filtration of the desired form, let "-" denote images in $R /(0: x)$. By Corollary 5.2, $\left\{\bar{x}_{\lambda} \mid \lambda \in \Lambda\right\} \subseteq R /(0: x)$ is a quadratic sequence. By the induction hypothesis on $n$, there exists a filtration of $\bar{R} / \bar{X}^{n-1}$ in which the quotients are cyclic $\bar{R}$-modules whose annihilators are related ideals of the quadratic sequence $\left\{\bar{x}_{\lambda} \mid \lambda \in \Lambda\right\}$. But the preimage in $R$ of an ideal in $\bar{R}$ related to $\left\{\bar{x}_{\lambda} \mid \lambda \in \Lambda\right\}$ is related to $\left\{x_{\lambda} \mid \lambda \in \Lambda\right\}$, as we now show. Clearly, every such preimage is of the form $\left(\left(X_{\Sigma}+(0: x)\right): x_{\lambda}\right)+X$ where $\Sigma$ is an ideal of $\Lambda$ and $\lambda$ is an element of $\Lambda$ that is just above $\Sigma$. Let $\Omega$ be an ideal of $\Lambda$ such that $(0: x) \cap X \subseteq X_{\Omega}$ and $x X_{\Omega}=0$; to see that such an ideal $\Omega$ exists, let $\Sigma$ be the empty subset and $\lambda=\alpha$ in the conditions of Definition 3.3 of a quadratic sequence. It suffices to prove the claim that $\left(\left(X_{\Sigma}+(0: x)\right): x_{\lambda}\right)=\left(X_{\Sigma \cup \Omega}: x_{\lambda}\right)$, for then the preimage under consideration will equal $\left(X_{\Sigma \cup \Omega}: x_{\lambda}\right)+X$ which is clearly related to $\left\{x_{\lambda} \mid \lambda \in \Lambda\right\}$ since $\lambda$ is inside or just above $\Sigma \cup \Omega$. We now prove the claim. It is a consequence of the general identity $(\mathbf{A}: \mathbf{B})=(\mathbf{A} \cap \mathbf{B}: \mathbf{B})$ for ideals $\mathbf{A}$ and $\mathbf{B}$ of a ring that $\left(\left(X_{\Sigma}+(0: x)\right): x_{\lambda}\right)=\left(\left(\left(X_{\Sigma}+(0: x)\right) \cap\left(x_{\lambda}\right)\right): x_{\lambda}\right)$. But, since $\left(X_{\Sigma}+(0: x)\right) \cap X$ is contained in $X_{\Sigma}+(0: x)$ and contains $\left(\left(X_{\Sigma}+(0: x)\right) \cap\left(x_{\lambda}\right)\right.$, this forces $\left(\left(X_{\Sigma}+(0: x)\right): x_{\lambda}\right)=\left(\left(\left(X_{\Sigma}+(0: x)\right) \cap X\right): x_{\lambda}\right)$. It is therefore enough to show that $\left(X_{\Sigma}+(0: x)\right) \cap X=X_{\Sigma \cup \Omega}$. It follows from the general identity $(A+B) \cap C=A+(B \cap C)$ for submodules of a module satisfying $A \subseteq C$, we get $\left(X_{\Sigma}+(0: x)\right) \cap X=X_{\Sigma}+((0: x) \cap X)$. But, since $(0: x) \cap X \subseteq X_{\Omega}$ and $x X_{\Omega}=0$, we have $(0: x) \cap X=X_{\Omega}$. Thus $\left(X_{\Sigma}+(0: x)\right) \cap X=X_{\Sigma}+X_{\Omega}$, and the latter clearly equals $X_{\Sigma \cup \Omega}$. This finishes the proof of the claim and also that of the theorem.

Corollary 5.5. Let $I$ be an ideal of a Noetherian ring $R$, and $\left\{x_{\lambda} \mid \lambda \in \Lambda\right\}$ be $a$ set of elements indexed by a finite poset $\Lambda$. Let $X$ denote the ideal $\left(x_{\lambda} \mid \lambda \in \Lambda\right)$. If $\left\{x_{\lambda} \mid \lambda \in \Lambda\right\} \subseteq R$ is a quadratic sequence with respect to the ideal $I$, and if $I^{k-1} / I^{k}$ is a free $R / I$-module for every positive integer $k$, then, for any positive integer $n$ and any ideal $\mathbf{p}$ of $R$,

$$
\operatorname{depth}\left(\mathbf{p}, \frac{R}{(I+X)^{n}}\right) \geq \min _{(\Sigma, \lambda)} \operatorname{depth}\left(\mathbf{p}, \frac{R}{\left(\left(I, X_{\Sigma}\right): x_{\lambda}\right)+X}\right),
$$

where the minimum is taken over all pairs $(\Sigma, \lambda)$ such that $\Sigma$ is an ideal of $\Lambda$ and $\lambda$ is an element of $\Lambda$ that is (inside or) just above $\Sigma$.

To prove this corollary, we use Transitivity of Depth, which was proved by Huneke in [H1]. Actually, we use the following slightly improved version of transitivity of depth proved in $[R]$.

Theorem 5.6 (Transitivity of Depth). Let $I$ and $X$ be ideals of a Noetherian ring $R$ and $n$ be a positive integer. If $I^{k-1} / I^{k}$ are free $R / I$-modules and $I \cap X^{l} \subseteq I X^{l-1}$ whenever $1 \leq k, l \leq n$, then, for any ideal $\mathbf{p}$ of $R$,

$$
\operatorname{depth}\left(\mathbf{p}, \frac{R}{(I+X)^{n}}\right) \geq \min _{1 \leq k \leq n} \operatorname{depth}\left(\mathbf{p}, \frac{R}{I+X^{k}}\right) \text {. }
$$

We also use the following two elementary results.

Lemma 5.7 [H1, Lemma 3.4]. Let $I$ and $X$ be ideals of a ring $R$ and let $n$ and $k$ be positive integers. If $I^{k-1} / I^{k}$ is a free $R / I$-module and $I \cap X^{n} \subseteq I X^{n-1}$, then $I^{k} \cap I^{k-1} X^{n} \subseteq I^{k} X^{n-1}$. 
Proposition 5.8 [H1, Lemma 3.2]. If $M=M_{0} \supseteq M_{1} \supseteq \cdots \supseteq M_{n}=0$ is a filtration of a finitely generated module $M$ over a Noetherian ring, then, for any ideal $\mathbf{p}$,

$$
\operatorname{depth}(\mathbf{p}, M) \geq \min _{0 \leq k \leq n-1} \operatorname{depth}\left(\mathbf{p}, \frac{M_{k}}{M_{k+1}}\right) .
$$

Proof of Corollary 5.5. Applying Corollary 3.7, we conclude that $I \cap X^{d} \subseteq I X^{d-1}$ for all $d \geq 1$. Lemma 5.7 enables us to apply Transitivity of Depth. We get

$$
\operatorname{depth}\left(\mathbf{p}, \frac{R}{(I+X)^{n}}\right) \geq \min _{1 \leq k \leq n} \operatorname{depth}\left(\mathbf{p}, \frac{R}{I+X^{k}}\right) .
$$

But, from the theorem we just proved, $R /\left(I+X^{k}\right)$ has a filtration in which the quotients are related ideals of the quadratic sequence $\left\{\bar{x}_{\lambda} \mid \lambda \in \Lambda\right\} \subseteq R / I$. From Proposition 5.8, we conclude that, for each $k$,

$$
\operatorname{depth}\left(\mathbf{p}, \frac{R}{I+X^{k}}\right) \geq \min _{(\Sigma, \lambda)} \operatorname{depth}\left(\mathbf{p}, \frac{R}{\left(\left(I, X_{\Sigma}\right): x_{\lambda}\right)+X}\right) .
$$

Corollary 5.9. Let $R$ be a Noetherian ring, $\left\{x_{\lambda} \mid \lambda \in \Lambda\right\} \subseteq R$ be a quadratic sequence indexed by a finite poset $\Lambda$, and let $X$ be the ideal $\left(x_{\lambda} \mid \lambda \in \Lambda\right)$. Assume that there exists a minimal element $\theta$ of $\Lambda$ such that $x_{\theta}$ is not a zero-divisor. Let $\mathbf{p}$ be any ideal of $R$, and let $s$ be the integer defined by

$$
s=\min _{(\Sigma, \lambda)} \operatorname{depth}\left(\mathbf{p}, \frac{R}{\left(X_{\Sigma}: x_{\lambda}\right)+X}\right),
$$

where the minimum is taken over all pairs $(\Sigma, \lambda)$ such that $\Sigma$ is an ideal of $\Lambda$ and $\lambda$ is an element of $\Lambda$ that is (inside or) just above $\Sigma$. Then, if $\operatorname{depth}\left(\mathbf{p}, R / X^{n}\right)=s$ for some positive integer $n$, then $\operatorname{depth}\left(\mathbf{p}, R / X^{m}\right)=s$ for every integer $m \geq n$.

Proof. Clearly we may assume $m=N+1$. Write $x$ instead of $x_{\theta}$. Let $J=$ $\left(x_{\lambda} \mid \lambda \in \Lambda, \lambda \neq \theta\right)$. Then, since $\left\{x_{\lambda} \mid \lambda \in \Lambda \backslash\{\theta\}\right\}$ is a quadratic sequence with respect to the ideal $(x)$ (see Remark 3.4), the second statement of Corollary 3.7 gives $(x) \cap X^{n+1}=(x) X^{n}$. Thus we have

$$
\frac{\left(x, J^{n+1}\right)}{X^{n+1}} \cong \frac{(x)}{(x) \cap\left(X^{n+1}\right)}=\frac{(x)}{(x) X^{n}} \cong \frac{R}{X^{n}},
$$

where the first isomorphism is a special case of the general isomorphism $(A+B) / B \cong A /(A \cap B)$ of submodules, and the last isomorphism follows from our hypothesis that $x$ in not a zero-divisor. This gives us the short exact sequence

$$
0 \rightarrow \frac{R}{X^{n}} \rightarrow \frac{R}{X^{n+1}} \rightarrow \frac{R}{\left(x, J^{n+1}\right)} \rightarrow 0 .
$$

It follows from the previous Corollary 5.5 that $\operatorname{depth}\left(\mathbf{p}, R /\left(x, J^{n+1}\right)\right) \geq s$ : let $I=(x)$ and notice that the right side of the inequality in the conclusion of the previous corollary is at least $s$. By our hypothesis we have $\operatorname{depth}\left(\mathbf{p}, R / X^{n}\right) \geq s$. It is now clear from the long exact sequence on $\operatorname{Ext}_{R}(R / \mathbf{p}, \cdot)$ induced by the above exact sequence that $\operatorname{depth}\left(\mathbf{p}, R / X^{n+1}\right)=s$.

Corollary 5.10. Let $R, \Lambda, X$, and $\theta$ be as in the above corollary. Then $\operatorname{Ass}(R / X) \subseteq \operatorname{Ass}\left(R / X^{2}\right) \subseteq \cdots$.

Proof. As in the previous corollary, $0 \rightarrow R / X^{n} \rightarrow R / X^{n+1}$ is exact. 


\section{A NoETHERIAN LOCAL RING NEED NOT BE BUCHSBAUM EVEN IF EVERY PARAMETER IDEAL IS GENERATED BY A $d$-SEQUENCE}

Motivation for the following example comes from Huneke's characterization of local Buchsbaum rings (see [H1, Theorem 1.7; SV1, Chapter I, Proposition 1.17]: a Noetherian local ring is Buchsbaum if and only if every system of parameters is a $d$-sequence. (There is a minor error in the proof of [ $\mathrm{H} 1$, Theorem 1.7] which is corrected in [R].) Let $R$ denote the subring $k\left[x, x y, y^{2}, y^{5}\right]$ of the polynomial ring $k[x, y]$ in two indeterminates over a field $k$. Let $\mathbf{M}$ denote the homogeneous maximal ideal $\left(x, x y, y^{2}, y^{5}\right)$ of $R$. We prove that the two dimensional Noetherian local domain $R_{\mathbf{M}}$ obtained by localizing $R$ at $\mathbf{M}$ is not Buchsbaum but every ideal of $R_{\mathbf{M}}$ generated by a system of parameters is even generated by a $d$-sequence.

Elements $a, b$ of a domain form a $d$-sequence if and only if $((a): b)=$ $\left((a): b^{2}\right)$ (by definition). To show that $R_{\mathbf{M}}$ is not Buchsbaum, we show that the system of parameters $x, y^{2}$ is not a $d$-sequence. This will suffice, for every system of parameters of a local Buchsbaum ring must be a $d$-sequence. In $R$, the sequence $x, y^{2}$ is not a $d$-sequence, since $x y$ belongs to the ideal $\left((x): y^{4}\right)$ but not to $\left((x): y^{2}\right)$. After passing to $R_{\mathbf{M}}$, the element $x y$ still belongs to $\left((x): y^{4}\right)$, since this ideal is obtained by extending the same colon ideal from $R$. To see that $x y^{3}$ does not belong to $(x)$ even after localization, notice that, if it did, then there would exist an element $a$ of $R$ not belonging to $\mathbf{M}$ (that is, $a$ has nonzero constant term) such that, back in the ring $R, a x y^{3}$ belongs to $(x)$. But that would be absurd, since no element of the ideal $(x)$ of $R$ can have a nonzero coefficient for $x y^{3}$.

To show that every parameter ideal of $R_{\mathrm{M}}$ is generated by a $d$-sequence, we first prove a proposition.

Proposition 6.1. Let $a, b$ be elements of $R=k\left[x, x y, y^{2}, y^{5}\right]$ that do not form a $d$-sequence and such that the ideal they generate has height 2 Then ay belongs to $R$ and, in fact, to $\left((a): b^{2}\right)$ but not to $((a): b)$, and the element $b$ after modification by multiplication by a nonzero element of the field $k$ has the form $y^{2}+b^{\prime}$, where $b^{\prime}$ is an element of $R$. In particular, the elements $b, a$ form a d-sequence.

Proof. To see that the second assertion follows from the first, suppose that $a, b$ is not a $d$-sequence. Then, by the first assertion, $b$ has the form $y^{2}+b^{\prime}$, which implies that $b y$ cannot belong to $R$. Hence, by the first assertion, $b, a$ must be a $d$-sequence.

To prove the first statement, let $c$ be an element of $\left((a): b^{2}\right)$ that does not belong to $((a): b)$. Since an ideal of height 2 of $R$ still has height 2 after extension to $S=k[x, y]$, the elements $a, b$ generate an ideal of height 2 in $S$. Since $S$ is Cohen-Macaulay, they form a regular sequence in $S$. Since $c b^{2}$ belongs to $a R$ (and hence also to $a S$ ) and $b^{2} \notin a S$, it follows that $c$ belongs to $a S$. Write

$$
c=\left(\alpha y+\beta y^{3}+c^{\prime}\right) \cdot a,
$$

where $\alpha$ and $\beta$ are elements of the field $k$ and $c^{\prime}$ belongs to $R$. Since $y^{3}$ multiplies the maximal ideal $\mathbf{M}$ of $R$ into $R$, it follows that $\left(\beta y^{3}+c^{\prime}\right) a$ belongs to $R$. Moreover, it belongs to $((a): b)$, since $\left(\beta y^{3}+c^{\prime}\right) b$ is in $R$. Since $c$ was chosen not to belong to $((a): b)$, this forces $\alpha$ to be nonzero. 
Note that modifying $c$ by subtracting an element of $((a): b)$ from it does not change its status as an element of $\left((a): b^{2}\right) \backslash((a): b)$. Subtracting $\left(\beta y^{3}+c^{\prime}\right) a$ from $c$ and multiplying by the inverse of $\alpha$, we get $a y$.

Now write $b=\gamma y^{2}+b^{\prime}$, where $\gamma$ is an element of the field $k$ and $b^{\prime}$ is an element of $\mathbf{M}$ having coefficient of $y^{2}$ equal to zero. Since $y$ multiplies $b^{\prime}$ into $R$, it follows that $b^{\prime} a y$ belongs to $a R$. If $\gamma$ were equal to zero, this would force $a y \in((a): b)$, a contradiction. Thus $\gamma$ is nonzero, and multiplying $b$ by the inverse of $\gamma$, we see that $b$ has the desired form.

Let $I$ denote the contraction to $R$ of an arbitrarily fixed parameter ideal of $R_{\mathbf{M}}$. Our goal is to show that the parameter ideal, which is nothing but the extension $I_{\mathbf{M}}$ of $I$ to $R_{\mathbf{M}}$, is generated by a $d$-sequence. There exist elements $a, b$ in $I$ whose images in $R_{\mathbf{M}}$ generate $I_{\mathbf{M}}$. We claim that these elements may be chosen such that they generate an ideal of height 2 . Given this claim, we conclude, using the above proposition, that either $a, b$ or $b, a$ is a $d$ sequence. Since a $d$-sequence stays a $d$-sequence in any localization, it follows that $I_{\mathbf{M}}$ is generated by a $d$-sequence. To prove the claim, suppose that the elements $a, b$ generate an ideal of height one. Modify $b$ by adding to it an element $c$ that belongs to $\mathbf{M} I$ and to every minimal prime over $(a)$ that does not contain $b$ but not to any minimal prime over $(a)$ that also contains $b$; such an element $c$ exists by prime avoidance, since $\mathbf{M} I$ is primary to $\mathbf{M}$ and therefore not contained in any prime of height 1 . The new $a, b$ generate an ideal of height 2 , and their images in $R_{\mathbf{M}}$ generate $I_{\mathbf{M}}$ by Nakayama since, by virtue of $c \in \mathbf{M} I$, the $R_{\mathbf{M}} / \mathbf{M} R_{\mathbf{M}}$ vector subspace of $I_{\mathbf{M}} / \mathbf{M} I_{\mathbf{M}}$ generated by the images of $a, b$ remains unaltered.

\section{A CRITERION FOR EQUALITY IN BURCH'S INEQUALITY}

In this section, we extend to the case of quadratic sequences a theorem of Huneke [H1, Theorem 4.1], which gives a sufficient criterion for equality to hold in Burch's inequality for an ideal generated by a $d$-sequence. Our proof is simpler than Huneke's proof for $d$-sequences. It also shows that the hypothesis of Huneke's theorem can be weakened. Herzog, Simis, and Vasconcelos have improved this theorem in another direction (see [HSV1, Theorem 5.12]).

Definition 7.1. The analytic spread of an ideal $I$ of a Noetherian local ring $R$ with maximal idea $\mathbf{M}$-the standard notation for which is $l(I)$-is the Krull dimension of the fiber over $\mathbf{M}$ in the Rees ring of $I$. Symbolically, we have

$$
l(I)=\operatorname{dim}\left(\frac{R[I t]}{\mathbf{M}[I t]}\right)=\operatorname{dim}\left(\frac{R}{\mathbf{M}} \oplus \frac{I}{\mathbf{M} I} \oplus \frac{I^{2}}{\mathbf{M} I^{2}} \oplus \cdots\right) .
$$

The analytic spread of an ideal of a local ring is bounded above by the number of generators of the ideal; this is immediate from the definition since the Rees ring (respectively, the fiber ring) is a homomorphic image of the polynomial ring over $R$ (respectively, over the residue field) in as many indeterminates as there are generators for the ideal. If an ideal is generated by analytically independent elements, then its analytic spread equals their number, since (by definition of analytic independence) the map from the polynomial ring over the residue field onto the fiber ring defined using these elements as generators for the ideal is an isomorphism. 
Let $I$ be an ideal of a local ring $R$. Burch $[\mathrm{Bu}]$ showed that

$$
l(I) \leq \operatorname{dim}(R)-\inf _{m \geq 1} \operatorname{depth}\left(R / I^{m}\right) .
$$

Brodmann $[\mathrm{Br}]$ improved this to

$$
l(I) \leq \operatorname{dim}(R)-\lim _{m \geq 1} \operatorname{depth}\left(R / I^{m}\right) .
$$

Note that if equality holds in Burch's inequality, then equality holds also in Brodmann's inequality, and $\inf _{m \geq 1} \operatorname{depth}\left(R / I^{m}\right)=\lim _{m \geq 1} \operatorname{depth}\left(R / I^{m}\right)$. We recall some notation from $\S 1$ : given elements $x_{1}, \ldots, x_{n}$ of a ring, we denote the ideal $\left(x_{1}, \ldots, x_{n}\right)$ by $X$, the partial ideal $\left(x_{1}, \ldots, x_{i}\right)$ by $X_{i}$, and we let $X_{0}=0$.

Theorem 7.2. Let $\left\{x_{\lambda} \mid \lambda \in \Lambda\right\} \subseteq R$ be a quadratic sequence of a Noetherian local ring $R$. Assume that $\operatorname{depth}(R / X) \geq \operatorname{dim}(R)-l(X)$, that $\operatorname{depth}\left(R / X_{\Sigma}\right) \geq$ $\operatorname{dim}(R)-l(X)+1$ for every proper ideal $\Sigma \subsetneq \Lambda$, and that $\operatorname{depth}(R / X) \geq$ $\operatorname{dim}(R)-l(X)+1$ if there exists an ideal $\Sigma$ of $\Lambda$ and an element $\lambda \in \Lambda$ just above $\Sigma$ such that $\left(X_{\Sigma}: x_{\lambda}\right) \supseteq X$. Then, for the ideal $X$, equality holds in Burch's inequality, that is,

$$
l(X)=\operatorname{dim}(R)-\inf _{m \geq 1} \operatorname{depth}\left(R / X^{m}\right) .
$$

Proof. In view of Burch's inequality, it suffices to show that $\operatorname{dim}(R)-l(X) \leq$ $\operatorname{depth}\left(R / X^{m}\right)$ for every positive integer $m$. But by Theorem 5.4 , there exists a filtration of the $R$-module $R / X^{m}$ (for every $m$ ) in which the quotients are cyclic modules whose annihilators are related ideals of the quadratic sequence. Recall that the depth of a finitely generated module $M$ over a local ring $R$ is equal to the least integer $j$ such that $\operatorname{Ext}_{R}^{j}(R / \mathbf{M}, M) \neq 0$, where $\mathbf{M}$ denotes the maximal ideal of $R$. It follows from the elementary Proposition 5.8 that

$$
\operatorname{depth}\left(\frac{R}{X^{m}}\right) \geq \inf _{(\Sigma, \lambda)} \operatorname{depth}\left(\frac{R}{\left(X_{\Sigma}: x_{\lambda}\right)+X}\right),
$$

where $(\Sigma, \lambda)$ run over all pairs such that $\Sigma$ is an ideal of $\Lambda$ and $\lambda$ is an element just above $\Sigma$. Therefore it suffices to show that

$$
\operatorname{depth}\left(\frac{R}{\left(X_{\Sigma}: x_{\lambda}\right)+X}\right) \geq \operatorname{dim}(R)-l(X) \quad \forall \text { such pairs }(\Sigma, \lambda) .
$$

Fix such a pair $(\Sigma, \lambda)$. Let $\Theta$ be an ideal of $\Lambda$ such that $\left(X_{\Sigma}: x_{\lambda}\right) \cap X=X_{\Theta}$. Consider the long exact sequence on Ext induced by the short exact sequence

$$
0 \rightarrow \frac{\left(X_{\Sigma}: x_{\lambda}\right)+X}{X} \rightarrow \frac{R}{X} \rightarrow \frac{R}{\left(X_{\Sigma}: x_{\lambda}\right)+X} \rightarrow 0 .
$$

From this, it is enough to show that

$$
\operatorname{depth} \frac{\left(X_{\Sigma}: x_{\lambda}\right)+X}{X} \geq \operatorname{dim}(R)-l(X)+1 \text {. }
$$

Notice that

$$
\frac{\left(X_{\Sigma}: x_{\lambda}\right)+X}{X} \cong \frac{\left(X_{\Sigma}: x_{\lambda}\right)}{X \cap\left(X_{\Sigma}: x_{\lambda}\right)}=\frac{\left(X_{\Sigma}: x_{\lambda}\right)}{X_{\Theta}} .
$$

Consider the long exact sequence on Ext induced by the short exact sequence

$$
0 \rightarrow \frac{\left(X_{\Sigma}: x_{\lambda}\right)}{X_{\boldsymbol{\Theta}}} \rightarrow \frac{R}{X_{\boldsymbol{\Theta}}} \rightarrow \frac{R}{\left(X_{\Sigma}: x_{\lambda}\right)} \rightarrow 0 .
$$


Since $\operatorname{depth}\left(R / X_{\Theta}\right) \geq \operatorname{dim}(R)-l(X)+1$ by hypothesis, it is enough to show that

$$
\operatorname{depth} \frac{R}{\left(X_{\Sigma}: x_{\lambda}\right)} \geq \operatorname{dim}(R)-l(X) \text {. }
$$

But this follows from our hypothesis and long exact sequence on Ext induced by the short exact sequence

$$
0 \rightarrow \frac{R}{\left(X_{\Sigma}: x_{\lambda}\right)} \stackrel{x_{\lambda}}{\longrightarrow} \frac{R}{X_{\Sigma}} \rightarrow \frac{R}{\left(X_{\Sigma}, x_{\lambda}\right)} \rightarrow 0 ;
$$

note that $\Sigma \cup\{\lambda\}$ is an ideal of $\Lambda$.

For examples of $d$-sequences to which the above theorem can be applied, see $\S 4$ of [H1]. The hypothesis of Theorem 4.2 of [H1] seem to be much stronger than that of the theorem above; I am not, however, aware of an example of a $d$-sequence that illustrates this. In the case of general quadratic sequences, it is typically easier to verify directly that

$$
\operatorname{depth}\left(\frac{R}{\left(X_{\Sigma}: x_{\lambda}\right)+X}\right) \geq \operatorname{dim}(R)-l(X),
$$

for all pairs $(\Sigma, \lambda)$ such that $\lambda$ lies just above $\Sigma$; for example, see Proposition 2.3 of [MS].

\section{ACKNOWLEDGMENTS}

The author thanks Bill Heinzer, Craig Huneke, and Mark Johnson for helpful discussions, and the referee for a careful reading.

\section{REFERENCES}

[Br1] M. Brodmann, Asymptotic stability of $\operatorname{Ass}\left(M / I^{n} M\right)$, Proc. Amer. Math. Soc. 74 (1979), 16-18.

[Br2] The asymptotic nature of analytic spread, Math. Proc. Cambridge Philos. Soc. 86 (1979), 35-39.

[BST] W. Bruns, A. Simis, and N. V. Trung, Blow-up of straightening-closed ideals in ordinal Hodge algebras, Trans. Amer. Math. Soc. 326 (1991), 507-528.

[BV] W. Bruns and U. Vetter, Determinantal rings, Lecture Notes in Math., vol. 1327, SpringerVerlag, Berlin, Heidelberg, New York, 1988.

[Bu] L. Burch, Codimension and analytic spread, Proc. Cambridge Philos. Soc. 72 (1972), 369-373.

[C] D. Costa, Sequences of linear type, J. Algebra 94 (1985), 256-263.

[CN] R. Cowsik and M. V. Nori, On the fibres of blowing up, J. Indian Math. Soc. 40 (1976), 217-222.

[DEP] C. DeConcini, D. Eisenbud, and C. Procesi, Hodge algebras, Astérisque 91 (1982).

[Fi] M. Fiorentini, On relative regular sequences, J. Algebra 18 (1971), 384-389.

[HLS] W. Heinzer, D. Lantz, and K. Shah, The Ratliff-Rush ideals in a Noetherian ring, Comm. Algebra 20 (1992), 591-622.

[HSV1] J. Herzog, A. Simis, and W. Vasconcelos, Approximation complexes of blowing-up rings, J. Algebra 74 (1982), 466-493.

[HSV2] - Approximation complexes of blowing-up rings. II, J. Algebra 82 (1983), 53-83.

[HuH1] S. Huckaba and C. Huneke, Powers of ideals having small analytic deviation, Amer. J. Math. 114 (1992), 367-403.

[HuH2] _ Rees algebras of ideals having small analytic deviation, preprint, 1990. 
[H1] C. Huneke, The theory of $d$-sequences and powers of ideals, Adv. Math. 46 (1982), 249-279.

[H2] _- Powers of ideals generated by weak d-sequences, J. Algebra 68 (1981), 471-509.

[H3] - On the symmetric and Rees algebra of an ideal generated by a $d$-sequence, J. Algebra 62 (1980), 268-275.

[H4] _ Determinantal ideals of linear type, Arch. Math. (Basel) 47 (1986), 324-329.

[H5] - Symbolic powers and weak d-sequences, Lecture Notes in Pure and Appl. Math., vol. 68, Dekker, New York, 1982, pp. 173-199.

[H6] _ The Koszul homology of an ideal, Adv. Math. 56 (1985), 295-318.

[HU] C. Huneke and B. Ulrich, Residual intersections, J. Reine Angew. Math. 390 (1988), 1-20.

[MS] M. Morales and A. Simis, Symbolic powers of monomial curves in $\mathbb{P}^{3}$ lying on $x y-z w=$ 0, Prépublication de l'Institut Fourier, no. 162, 1990.

[NR] D. Northcott and D. Rees, Reductions of ideals in local rings, Proc. Cambridge Philos. Soc. 50 (1954), 145-158.

[R] K. N. Raghavan, Uniform annihilation of local cohomology and powers of ideals generated by quadratic sequences, Purdue Univ. Thesis, 1991.

[R1] $\quad$ A simple proof that ideals generated by $d$-sequences are of linear type, Comm. Algebra 19 (1991), 2827-2831.

[S] P. Schenzel, Examples of Gorenstein domains and symbolic powers of monomial space curves, preprint, 1989.

[SV] J. Stückrad and W. Vogel, Towards a theory of Buchsbaum singularities, Amer. J. Math. 100 (1978), 727-746.

[SV1] _ Buchsbaum rings and applications, Springer-Verlag, Berlin and New York, 1986.

[V] G. Valla, On the symmetric and Rees algebras of an ideal, Manuscripta Math. 30 (1980), 239-255.

[Vas] W. Vasconcelos, Koszul homology and the structure of low codimension Cohen-Macaulay ideals, Trans. Amer. Math. Soc. 301 (1987), 591-613.

Department of Mathematics, Michigan State University, East Lansing, Michigan 48824-1027

Current address: School of Mathematics, SPIC Science Foundation, 92 G. N. Chetty Road, T. Nagar, Madras 600 017, India

E-mail address: knr@ssf.ernet.in 